\title{
Review of: "Glycation damage to organelles and their DNA increases during maize seedling development"
}

\author{
Bartolome Vilanova CAnet \\ 1 Universitat de les Illes Balears
}

Potential competing interests: The author(s) declared that no potential competing interests exist.

The paper of Tripathi et al. study the impact of glycation in seedlings grown in the dark and in the light.The authors mainly analysed glycation damage and glycation defense in plant organelles. They have reported changes in the levels of glycation adducts in proteins and DNA, as well as MGO and DJ-1, in organelles at three stages of maize development. In general, the data are consistent and interesting and are well supported by biochemical analysis. It is a valuable contribution to maize seedling development.

On the other hand, the formation of methyl glyoxal in plants is not well described in the paper. Authors should indicate that MG is mainly formed by the trace-level degradation of triosephosphates, glyceraldehyde-3-phosphate (GA3P) and dihydroxyacetonephosphate (DHAP) (Int J Mol Sci 2020, 21 (11), 3942). GA3P is an intermediate in photosynthesis, and GA3P and DHAP are intermediates in glycolysis, gluconeogenesis and glyceroneogenesis in lipid synthesis. 3-DG is formed by the enzymatic and non-enzymatic degradation of proteins glycated by glucose, that can be related to Figure 1 . 